\title{
Optimal Model-Based Control of Non-Viral siRNA Delivery
}

\author{
Elnaz Jamili and Vivek Dua* \\ Department of Chemical Engineering, Centre for Process Systems Engineering, University \\ College London, Torrington Place, London, WC1E 7JE, United Kingdom
}

\author{
*Corresponding author: Vivek Dua \\ Phone number: +44 (0) 2076790002 \\ E-mail address: v.dua@ucl.ac.uk
}

Short running title: Optimal Control of siRNA Delivery 


\begin{abstract}
Further quantitative understanding of the biological effects and mechanisms involved in cellular and intracellular delivery of nucleic acid materials is critical to produce clinical applications in the area of gene therapy. Several modeling approaches have been used in this field; however, a comprehensive approach that integrates all the key pharmacological issues into a holistic framework that is applicable for in vivo conditions is still lacking. This contribution presents a pharmacokinetic/pharmacodynamic model-based control study of non-viral siRNA delivery describing the dynamics of the delivery process and takes into account the main multi-objective optimization issues such as efficacy and toxicity, as well as the effect of uncertainty in cell doubling time. The methodology developed in this work is used to predict the optimal dosage injection rate and optimal intracellular exposure of siRNAs in order to improve pharmacological effects before cell division occurs. The present analysis successfully provides quantitative predictions of non-viral siRNA activity paving the path for further experimental work to probe more efficient delivery systems.
\end{abstract}

Key words: siRNA delivery; Pharmacokinetic/pharmacodynamic modeling; Optimal control; Cell division; Toxicity; Efficacy 


\section{Introduction}

Researchers in fields ranging from nanomedicine to gene therapy have investigated a broad range of nucleotide-based therapies. The RNA interference (RNAi) pathway is one of such methods which emerged in 1998 with the aim of gene silencing (Fire et al, 1998). The strategy of using synthetic small interfering RNA (siRNA) as a therapeutic agent has become a powerful tool for the post-transcriptional knockdown of defective genes in mammalian cells with the aim of treating severe diseases such as viral infection and cancer. While optimization of siRNA design along with chemical modifications can reduce off-target effects and improve the stability of siRNAs, safe and efficient delivery is still a key challenge in realizing the clinical potential of RNAi therapeutics. Non-viral nanocarrier formulations are effective delivery systems to improve the pharmacokinetic properties and maximize the siRNA cargo at its site of action, cytoplasm. However, several extracellular and intracellular barriers as well as different interactions between nanocarriers and biological systems can restrict the efficient delivery of therapeutic agents (Bumcrot et al., 2006; Kanasty et al., 2012; Semple et al., 2010; Wang et al., 2010; Whitehead et al., 2009). Significant efforts have been made to develop non-viral delivery carriers and a number of recent studies have pointed to the design and structure of various types of non-viral systems (reviewed in detail by: Videira et al., 2014; Williford et al., 2014; Yin et al., 2014). In this work, a novel type of cationic lipidbased nanocarrier (NC), SPANosomes (SP) formulation, which was developed and evaluated by Zhou et al. (2012), is investigated for modeling and control purposes.

Advanced modeling and control techniques are not only helpful for understanding the dynamics of a gene delivery process, but also are essential for optimizing and controlling the delivery amount, timing, and speed in an effective and safe way. The area of modeling for

gene delivery systems has emerged since Ledley and Ledley (1994) developed a 
multicompartment and numerical model for studying the kinetics of cellular processes. Since that time, researchers have made considerable efforts in the development of mathematical and computational models for understanding the cellular transport processes (Schwake et al., 2010; Zhou et al., 2007). Most authors (Banks et al., 2003; Ledley and Ledley, 1994; Varga et al., 2001, 2005) developed mathematical modeling based on the concept of mass action kinetic model to investigate the critical steps involved in the gene delivery process. Although the exact mechanisms of the biological effects and the transfection process are not fully understood, further development of mathematical and computational methodologies has provided insights into the process. Such methodologies include quantitative structure-activity relationship (QSAR) modeling strategy (Horobin and Weissig, 2005), stochastic simulations (Dinh et al., 2007), semi-mechanistic model of transgene expression (Berraondo et al., 2009), mechanistic spatiotemporal and stochastic model of DNA delivery (Jandt et al., 2011), and telecommunication model (Martin et al., 2015). However, no effort has been made to develop a holistic framework that is applicable for in vivo gene therapy, and provides a model-based decision-making platform taking into account the main multi-objective optimization issues, which consequently forms the main objective of this paper. Table I summarizes the key limitations of previous computational studies.

A literature survey revealed that while a number of research had been done on model-based control of drug delivery (Dua et al., 2010), very little thought had been given to the development of control strategies for gene delivery systems (Dua, 2012; Jamili and Dua, 2016; Ma and Zhang, 2009). This paper presents the mathematical modeling, simulation and control of the dynamic process of non-viral siRNA delivery. We developed an integrated computational framework that is capable of modeling simultaneously intracellular trafficking of siRNAs and biological effects such as efficacy and toxicity while taking into account the effect of possible uncertainties in cell doubling time. The proposed model follows a 
compartmentalization approach. Compartmental modeling frameworks (Banks et al., 2003) are amenable for control purposes (Parker and Doyle, 2001), an advantage over detailed models (Dinh et al., 2007). Pharmacokinetic/pharmacodynamic (PK/PD) modeling approach can be valuable in the optimization and rational development of non-viral gene-based therapies (Parra-Guillen et al., 2010).

In the present work, an integrated PK/PD modeling platform for both efficacy and safety was developed based on in vitro experimental analysis to provide quantitative understanding of non-viral siRNA delivery. The developed PK/PD models were then used for an optimal control formulation that is set up to optimize siRNA delivery, which is applicable for in vivo conditions. The proposed modeling and control approach effectively computed an optimal infusion rate of siRNA therapeutics while simultaneously considering key pharmacological issues. Unlike many previous studies that partially describe the complexity of gene delivery processes (Banks et al., 2003; Ledley and Ledley, 1994; Varga et al., 2001, 2005), the developed mathematical modeling and control framework in this research study provide an effective trade-off decision-making platform for siRNA delivery to take into account the main multi-objective optimization issues such as efficacy, toxicity, and the influence of uncertainty in cell division time. Effect of variations in cell doubling time was explored in the course of model assembly by incorporating time constraints into the optimization problem to achieve maximum desired effects before cell division occurs. A number of dynamic simulations were also performed comparing continuous infusions with bolus injections of siRNA therapeutics. According to the obtained results, a single bolus administration of therapeutic agents is not optimal for obtaining a persistent reduction in expression of defective genes. Therefore, maximum therapeutic effect with minimal toxicity was manifested with an optimal continuous infusion of siRNAs over time, before cell division takes place. 


\section{Methods}

\subsection{Mathematical Modeling of siRNA Delivery}

A pharmacokinetic model was developed based upon the level of available experimental data to represent intracellular transport processes responsible for delivery of siRNA during in vitro cell transfection. The PK model was then modified to include the infusion rate of siRNAs for in vivo delivery. A pharmacodynamic model was constructed consistent with published data to study the relationship between siRNA concentration and pharmacological effects. The developed PK model, coupled with the PD model, provided an integrated PK/PD modeling platform that was used for a multi-objective optimization framework in the presence of different practical constraints in order to obtain an optimal siRNA delivery infusion profile for in vivo conditions.

Development of high-fidelity mathematical models involves parameter estimation in which the objective is to minimize the summed square of the difference between the set of experimental data and the model predictions (Englezos and Kalogerakis, 2001). The focus of this paper is on a gene delivery system that follows pharmacokinetic modeling approach involving ordinary differential equations (ODEs). Parameter estimation of such systems requires solving a dynamic optimization problem. In this work, a simultaneous parameter estimation approach was performed using Artificial Neural Network (ANN) approximations (Dua and Dua, 2012). Orthogonal Collocation on Finite Elements (OCFE) was then used as a solution scheme for the full discretization of the dynamic models in the gene delivery optimal control problem. For the purpose of comparison, the dynamic simulations were validated using a well-known fourth order Runge-Kutta (RK4) scheme and the meshless ANN framework. All the optimization problems were formulated as nonlinear programming (NLP) problems and solved using the General Algebraic Modeling System (GAMS) (Brooke et al., 1998). 


\subsection{Experimental Data}

In an experimental study by Zhou et al. (2013), SK Hep-1 cells (human hepatocellular cell line) were transfected with siRNA/NC complexes, and the overall cellular and cytoplasm exposure of siRNA were reported over a 24-hour period as a function of time. The authors analyzed the cellular pharmacokinetics of siRNA for a novel type of nanocarrier, SPANosomes (SP) formulation. They also reported the gene silencing activity and cytotoxicity of siRNA/NC complexes. In the current computational work, the published experimental data were used to develop an integrated PK/PD model that describes the kinetic pathways of nanocarrier-mediated disposition of siRNA so as to effectively predicts the siRNA exposure to its site of action while improving pharmacological effects.

\subsection{Pharmacokinetic Modeling}

Mathematical representations of the PK model were used for quantitative evaluation of in vitro transfection. There are several biological barriers for NC-mediated siRNA delivery to reach their intended targets. The focus of this work is on intracellular barriers where the developed PK compartmental model, which is based upon the available experimental data, includes two compartments: the endosome and the cell cytoplasm. Figure 1 shows the model structure in which siRNA therapeutics can be transferred in one direction across the barriers. Despite the advantages of complex compartmental model structures such as better predictive performance, their use in control purposes would be limited due to their size (Parker and Doyle, 2001). In this work, the developed compartmental representation of siRNA delivery uses minimum number of compartments and unknown parameters to accurately capture the available experimental data and replicate the behavior of siRNA/NCs in vitro. The following system of ODEs, resulting from mass balances, represents the two-compartment model of siRNA pharmacokinetics from in vitro experimental data. The compartmental model, which indicates the changes in the amount of siRNA inside the endosome and cytoplasm over time, 
is given by:

$\frac{d}{d t} E(t)=-\theta_{1} E(t)$

$\frac{d}{d t} C(t)=\theta_{1} E(t)-\theta_{2} C(t)$

where $E(n M)$ and $C(n M)$ are state variables and represent the siRNA concentration in the endosome and in the cytoplasm, respectively. $\theta_{1}\left(h^{-1}\right)$ and $\theta_{2}\left(h^{-1}\right)$ are rate constants controlling the transport of siRNA from the endosome to the cytoplasm $\left(\theta_{1}\right)$ and trafficking through the cytoplasm in order to be loaded onto RNAi machinery $\left(\theta_{2}\right)$.

\subsection{Pharmacodynamic Modeling and Optimal Control of siRNA Delivery}

\subsubsection{Gene Silencing Activity}

In in vivo conditions, siRNA/NCs can be infused over a period of time (Fig. 1). So, Equation (1) is modified as follows:

$\frac{d}{d t} E(t)=-\theta_{1} E(t)+q(t)$

where $q(t)(n M / h)$ is the flow rate of the siRNA therapeutics infused. Incorporating the infusion rate into the PK model allows for the computation of optimal siRNA delivery profile in the presence of different practical constraints. In this work, the main objective is to minimize the total inhibitory effect over the time of therapy to achieve an efficient gene silencing by optimally infusing the siRNA/NCs at rate $q(t)$. The total siRNA concentration in the cell $\left(C_{\text {SiRNA }}, n M\right)$ is considered as a determinant for efficacy, so the following relationship is assumed:

$I(\%)=\left(I_{\max }+I_{0}\right)-I_{\max } \times \frac{C_{\text {siRNA }}}{C_{\text {siRNA }}+I C_{50}}$ 
where $I$ is the inhibitory effect, $I_{\max }(\%)$ represents the maximum inhibitory effect, $I_{0}(\%)$ is a baseline effect parameter when maximum therapeutics are present in the cell, and $I C_{50}(n M)$ is the siRNA concentration required to produce $50 \%$ of the maximum inhibitory effect. To estimate the pharmacodynamic parameters, the inhibitory effect model, Equation (4), was fitted to the relative gene expression values, which had been experimentally observed by Zhou et al. (2013).

\subsubsection{Cytotoxicity}

Cellular toxicity is investigated by considering a population of alive cells known as Cell Viability, which is controlled by two processes: production of cells and cell loss.

Cell Viability $=$ production of cells - cell loss

To describe the cytotoxic effects, the sigmoid Hill equation model has been modified to a composite $E_{\text {max }}$ model to include a no-drug response $\left(C V_{0}\right)$ and concentration effects over time. The following relationship was constructed such that the both processes of cell production and cell loss are functions of total siRNA concentration in the cell:

$$
C V(\%)=C V_{0}+\frac{G_{\max } \times C_{s i R N A}^{\gamma}}{G C_{50}{ }^{\gamma}+C_{\text {siRNA }}{ }^{\gamma}}-\frac{T_{\max } \times C_{\text {siRNA }}^{\gamma}}{T C_{50}{ }^{\gamma}+C_{\text {siRNA }}{ }^{\gamma}}
$$

where $C V$ denotes the Cell Viability indicating the percentage of alive cells during the therapy, $C V_{0}(\%)$ represents the initial percentage of alive cells, $G_{\max }(\%)$ is the maximum percent growth, $G C_{50}(n M)$ is the siRNA concentration required to produce $50 \%$ of the maximum percent growth, $T_{\max }(\%)$ denotes the maximum toxicity, $T C_{50}(n M)$ is the siRNA concentration required to produce $50 \%$ of the maximum toxicity, $\gamma$ is the power parameter to account for the curvature. The situation of combined drug action occurs when a single drug performs simultaneously at two different receptors (Gabrielsson and Weiner, 2010). The $C V$ model, Equation (6), represents a biphasic concentration-effect relationship 
involving two phases: phase A corresponded to the protective effect on cell viability (production process), whereas phase B reflected the cytotoxicity (loss process). The former phase is represented by the $G_{\max }$ term and the latter phase is represented by the $T_{\max }$ term. In this work, cell viability values, which had been experimentally observed by Zhou et al. (2013), were fitted to the developed $C V$ model in order to obtain the corresponding pharmacodynamic parameters.

\subsubsection{Optimal Control}

The siRNA delivery optimal control problem is formulated and solved subject to the system models and a set of constraints for computing an optimal infusion rate at optimal times. To this purpose, a multi-objective optimization framework is applied dealing with a number of objective functions to be optimized simultaneously. The siRNA delivery optimal control problem is of the following form:

$\min _{q(t)} T I E=\int_{t=0}^{t=t_{f}} I(t) d t=\Delta t \sum_{t=0}^{t=t_{f}} I(t)$

$\max _{q(t)} C V(t)$

subject to the system models and initial conditions; where TIE represents the Total Inhibitory Effect, and $t_{f}(h)$ is the final time at the end of the therapy. The first objective, minimization of TIE, aims to reduce undesirable gene expression, while the second objective, maximization of $C V(t)$, aims at minimizing cytotoxicity by preserving the life of the cells. The above multi-objective optimal control problem is reformulated as an $\epsilon$-constrained optimization problem (Clark and Westerberg, 1983), with the total inhibitory effect treated as the primary objective to be minimized, which is stated as follows: 
$\min _{q(t)} T I E=\int_{t=0}^{t=t_{f}} I(t) d t=\Delta t \sum_{t=0}^{t=t_{f}} I(t)$

subject to: $C V(t) \geq C V^{L O}$, Equations (2) - (4), (6), and initial conditions.

where $C V^{L O}(\%)$ is the minimum acceptable Cell Viability level. A number of case studies are presented in Section 3 to demonstrate the advantages of the proposed model-based optimal control framework for siRNA delivery. A conceptual block diagram representing a closed-loop model-based control scheme is shown in Figure 2.

\section{Results}

In the present work, we consider the following PD parameters, which were reported by Zhou et al. (2013), $I_{0}=5.2 \%$ and $I C_{50}=5.5 n M . I_{\max }$ was obtained as $94.8 \%$ by fitting the relative gene expression values to the inhibitory effect model given by Equation (4). Cell viability values were also fitted to the $C V$ model (Equation 6) to obtain the following PD parameters: $G_{\max }=21.2 \%, G C_{50}=15.4 \mathrm{nM}, T_{\max }=71.7 \%, T C_{50}=94.9 \mathrm{nM}$, and $\gamma=$ 7. The developed PK/PD models in this work were validated by comparing their predictions to experimental measurements, which is demonstrated in Supplementary Appendix as Supplemental Fig. S1 and S2. The schematic illustration of the composite $G_{\max } / T_{\max }$ model may also be found in Supplementary Appendix (Fig. S3), indicating that the estimated PD parameters in this study are in accordance with the system biology. We have also performed sensitivity analysis (Fig. S4) to determine the relative impact of each parameter on the $C V$ model output. The role of each parameter in the model was determined by varying only one parameter at a time while keeping all the other parameters constant, set at their estimated values. From our analysis, the maximum percent growth parameter, $G_{\max }$, turned out to be the most sensitive parameter. 
As cells were transfected for $4 h$ followed by $44 h$ incubation, a $48 h$ time frame $\left(t_{f}=48 h\right)$ was assumed for the simulation and optimization problems. Intracellular exposure of siRNA, gene silencing activity, and cytotoxicity resulting from two different delivery modes are compared and reported in Sections 3.1 and 3.2. PK/PD profiles were observed following a bolus administration of siRNA therapeutics and a continuous infusion over a period of time.

\subsection{Bolus Injection}

Single-dose bolus injections were simulated and the model was implemented in GAMS. Figure 3 shows the siRNA concentration in the cell, inhibitory effect, and cell viability as a function of time, after start of treatment with total injected doses of 80, 100, 150 and $250 \mathrm{nM}$. Time profile of siRNA concentration in the endosome and cytoplasm are shown in Figure 3a and $3 \mathrm{~b}$ respectively, when the therapeutic is administered as a bolus dose. The observed reduction of siRNA concentration over time for all four different doses in the endosome is due to the cellular distribution and irreversible elimination of the compound. siRNA therapeutics must be capable of escaping the endosome-lysosome degradation axis and releasing from their carriers to the cytoplasm so as to be loaded onto RNA-Induced Silencing Complex (RISC) (Wang et al., 2010).

Pharmacological responses depend on the total siRNA concentration in the cell, so minimum inhibitory effect can be achieved once a bolus dose of therapeutics is administered (Fig. 3c). The decrease in the total inhibitory effect appeared to be dose-dependent. Increasing the infusion from a low dose of siRNA therapeutics $(80 \mathrm{nM})$ to intermediate doses $(100 \mathrm{nM}$ and $150 \mathrm{nM})$, and finally to a high dose $(250 \mathrm{nM})$ will decrease the total inhibitory effect. For more details see Figure S5 in the Supplementary Appendix. According to Figure 3d, the reduction in cell viability is associated with high-dose bolus injections. No significant 
cytotoxicity was observed in the low injected dose $(80 \mathrm{nM})$; however, higher dose of siRNA therapeutics could decrease cell viability in a dose-dependent manner.

There is a rapid rise in the inhibitory effect profile while siRNA concentration is getting decreased over time (Fig. 3c). Therefore, a single bolus infusion of siRNAs resulted in a transient dose-dependent decrease in inhibitory effect that influences on the total inhibitory effect to become about 10 times greater for bolus injection, suggesting an optimal continuous siRNA infusion is more favorable delivery mode. The observed PK/PD profiles following a continuous infusion over a time frame of $48 h$ are reported and discussed in the next section.

Note that, the OCFE-based simulation results were validated by comparisons with ANN and RK4 simulation values and the results are shown in the Supplementary Appendix (Supplementary Fig. S6).

\subsection{Optimal Control of siRNA Delivery}

The siRNA delivery optimal control problem was solved for lower bound values of $50 \%$, $60 \%, 70 \%, 80 \%, 90 \%$ and $100 \%$ that were placed on cell viability. Figure 4 shows the multi-objective optimization results describing the trade-off between the minimum total inhibitory effect and lower bounds on cell viability. A relaxation on $C V^{L O}$ results in a decrease in the minimum total inhibitory effect that can be achieved (Fig. 5c), and an increase in the infusion rate (Fig. 5a). Typically, there is a conflict between efficacy and toxicity. Minimal inhibitory effect cannot be achieved without sacrificing the safety. According to the obtained results and comparing the six different case studies of various lower bounds, increasing the siRNA infusion rate would lead to a reduction in the inhibitory effect while increasing the risk of toxicity-induced cell death (Fig. 5). An optimal infusion rate was computed such that the total inhibitory effect was minimized and bounds on cell viability were respected. Figure 5a shows the optimal siRNA concentration that can be infused over a 
48-hour period of time to achieve maximum gene silencing activity (Fig. 5c) while maintaining cell viability at desirable levels (Fig. 5d).

The control optimization problem was solved subject to the constraints on the cell viability. Adding sensible bounds is necessary to ensure that minimum toxicity can be achieved, while still reaching maximum knockdown efficacy (Fig. 5d). According to Zhou et al. (2013), the production process is presumed to occur because low concentrations of siRNA/NC complexes can stimulate cell metabolic activity that leads to increase the apparent cell viability in comparison with the untreated group. However, the loss process could be due to the fact that the cells may die if the level of toxicity rises above a certain level. Undesirable effects such as toxicity result from the interactions between biological components and foreign materials such as siRNA molecules, gene carriers alone or in formulation with siRNAs (Kanasty et al., 2012; Lv et al., 2006; Nel et al., 2009). Here, the observed cytotoxicity is probably due to the surfactant activities of Span 80 or the other compositions of the SP formulation. Zhou et al. (2013) reported that the helper component in the SP formulation might make an important contribution to the cytotoxicity of the NCs. However, siRNA molecules can also elicit adverse biological effects include immune stimulation resulting in inflammatory responses and off-target silencing leading to toxicity (Kanasty et al., 2012; Wang et al., 2010; Xue et al., 2014). Figure 5b shows the exposure of siRNAs in the cytoplasm. siRNA therapeutics are first released in the endosome and then dispersed throughout the cytoplasm. As time passes, siRNA concentration in the endosome is shown to rise at a rapid pace suggesting that the infused siRNAs accumulate in the endosome upon their arrival (Data not shown). After infusing the genetic materials, a fraction of siRNAs is escaped from the endosome into the cytoplasm and gets distributed throughout the cytoplasm and finally transferred to the site of action in order to elicit their biological effects. 
Depending on the practical limitations of gene delivery devices, different constraints can be introduced into the optimization model. Two such examples are: (i) constraints on the process control variable, e.g. the maximum value that infusion can take, and (ii) the incremental change in infusion rate, $\Delta q(t)$, can be constrained between certain lower and upper bounds. In this section, a case study was first considered when $q$ was unconstrained to investigate the controlled siRNA delivery when practical limitations were not imposed. The siRNA delivery optimal control problem was also formulated and solved where practical limitations of gene therapy devices were imposed thereby an important constraint was introduced and incorporated into the control framework:

$0 \leq q(t) \leq q^{\max }$

where $q^{\max }$ represents an upper bound on the infusion rate indicating the maximum value that infusion can take during the therapy. In the presence of constraints on $q$, i.e. $q^{\max }=$ $30 \mathrm{nM} / \mathrm{h}$ or $q^{\max }=40 \mathrm{nM} / \mathrm{h}$, while lower bound of $100 \%$ on cell viability is respected, the results are shown in Figure 6. For both cases, a maximum allowable value of infusion takes place initially, which drops with time and then increases to a plateau of $21.85 \mathrm{nM} / \mathrm{h}$ for the rest of the therapy to achieve persistent gene silencing with minimum adverse side effects (Fig. 6).

The observed initial spike in the siRNA infusion rate in Figure 5a, which decreases rapidly in the first hour after delivery, reveals the need to introduce another important constraint into the formulation. So, incremental change in infusion rate, $\Delta q(t)$, is constrained between certain lower and upper bounds of $1 \mathrm{nM} / \mathrm{h}$, which is modelled as follows and the results are shown in Figure 7.

$\Delta q^{\min } \leq \Delta q(t) \leq \Delta q^{\max }$ 
This constraint was proposed to reduce problems associated with practical limitations of infusion devices in which the transfer of a large amount of therapeutics over a short period of time would be impossible. The observed results suggest that the system could be controlled in the presence of different constraints. When practical limitations are imposed, a high value of siRNA infusion takes place initially, which eventually decreases to a plateau, to obtain minimum total inhibitory effect over the therapy (Fig. 7).

\subsection{Incorporating Time Constraints}

The proposed model-based optimal control framework in the previous section addressed siRNA delivery to non-dividing cells. The aim of this section is to address the issues pertaining to the presence of constraints in the developed models to study the effects of mitosis and uncertainties in cell division time. A generic formulation for non-dividing conditions was presented in section 2.4 where constraints on process variables were incorporated into the siRNA delivery optimal control problem. Another critical constraint is on the time to take into account the cell multiplication so that the therapeutic effect is manifested before cell division takes place. To this purpose, the developed PK/PD models and control framework were used to incorporate the required constraints in order to deal with disruptions of cell proliferation. If the siRNAs inside the cytoplasm are loaded onto RNAi machinery and exert their therapeutic effects before the time of mitosis, the desired effects will remain in the newly formed cells. So, the length of the whole cell cycle should be considered in the formulation, as the site of action for siRNA therapeutics is the cytoplasm. According to research findings from literature (Ling et al., 2012), the doubling time for SK HEP-1 cells is approximately $25 h$, so optimal concentration of siRNA therapeutics must be delivered to the cytoplasm before cell division takes place. It is then possible to set an optimal control problem, by defining time constraints. According to Sandler et al. (2015), there is variability in cell cycle duration as different conditions could affect various stages of 
the cell cycle. For instance, in this study, low concentrations of siRNA/NC complexes can stimulate cell metabolic activity (Zhou et al., 2013) that could affect cell cycle in SK HEP-1 cells in comparison with the untreated group. To assess if uncertainties in doubling time affected the siRNA delivery process, a constrained optimization problem was developed and solved while considering different cell doubling time $\left(T_{d}\right)$ values of $4,10,15,20$ and $25 h$. Cell division greatly affects transfection (Martin et al., 2014), so the duration of therapy relies on the cell doubling time. Infusion must be completed before cell division takes place in order to achieve more efficient therapy. Therefore, siRNA therapeutics are assumed to be infused over the period of time required to obtain maximum desired effects, so the infusion is stopped at the doubling time while the algorithm considers the system performance for further two hours. These assumptions were performed for all case studies in this section and they can potentially be modified under different experimental conditions.

Figure 8 a shows the time profile of optimal siRNA infusion for different cell doubling times. As the doubling time increases, the amount of infusion increases. Longer transfection times require more siRNAs to keep the therapeutic effect at a desirable level. As it is signified in Figure 8a, a large amount of siRNAs can be infused at the start of treatment to get maximal knockdown while minimizing cytotoxicity. Minimum inhibitory effect is reached before cell division takes place. Once cells divide, siRNA infusion is discontinued and remains steady at $0 n M / h$ towards the end of the study resulting in increase in both inhibitory effect and cell viability (Fig. 8).

According to Zhou et al. (2013), only the portion of siRNA therapeutics that is released from the carriers into the cytoplasm is considered as determinants for gene silencing activity. The results in Figure $8 \mathrm{~b}$ suggest the optimal siRNA exposure at the target compartment, cytoplasm, for exerting maximum therapeutic efficacy over the therapy. Constraints on the $C V$ model were proposed to address issues associated with cytotoxicity. The cell viability is 
increased rapidly just after the first injection suggesting that low concentrations of siRNA/NC complexes stimulate cell metabolic activity leading to an increase in the apparent cell viability (Zhou et al., 2013). However, as the siRNA concentration in the cell (Fig. 8b) increases, cell viability decreases and remains bounded until cell division takes place (Fig. 8d).

From the observed results, it can be concluded that the model-based optimal control methodology provided a balance between efficacy and toxicity for siRNA delivery, while considering the effect of uncertainties in cell division time on intracellular transport.

The current findings have provided valuable information; however, challenges remain such as capability of infusion devices to deliver a large amount of therapeutics for different time frames. So, the developed model was reformulated as the control variable was constrained to investigate the behavior of the system over different time frames while simultaneously considering efficacy, toxicity, mitosis and the uncertainty in cell division time, which allows for representing siRNA delivery with wider scope. The optimal control results and the pharmacodynamic responses of this case study are presented in Figure 9 in which the maximum value that infusion can take is $40 \mathrm{nM} / \mathrm{h}$. In this case, the total inhibitory effect is higher than that of achieved for unconstrained $q$. Once the therapy is initiated, $q$ takes the maximum allowable value of $40 \mathrm{nM} / \mathrm{h}$, which then drops over the first hours of therapy. The siRNA infusion rate depends upon the transfection time periods. As the cell doubling time increases, longer transfection times are required, so more siRNA therapeutics must be infused to keep the inhibitory effect and the cell viability at desirable levels (Fig. 9).

Our novel application of the optimal control problem in siRNA delivery systems aims to simultaneously describe the intracellular transfection process and incorporate the main multiobjective optimization issues such as efficacy and toxicity, as well as uncertainties in cell 
doubling time. The developed integrated modeling platform can be simply adapted for a wide range of conditions such as different carriers and various practical limitations. Different scenarios were defined in this study and the corresponding results are available in the Supplementary Appendix (Supplementary Fig. S7, S8 and S9).

\section{Discussion}

An optimal delivery of siRNA-based therapeutics into the site of action is critical for the safety and efficacy of RNAi therapy for patients suffering from diseases that are associated with undesirable gene expression. This computational study begins with the development of an integrated PK/PD model, consistent with the level of available experimental data, demonstrating the time-concentration-effect relationship for siRNA/NC complexes. The siRNA delivery by non-viral nanocarriers was modelled as biochemical reactions illustrating the critical steps involved in the delivery. This is favourable as it allows us to study the mechanisms of siRNA delivery by determining the rate-limiting steps in NC-mediated delivery. From a kinetic point of view, the rate constants obtained from our quantitative analysis of in vitro experimental data (Supplementary Table S1), suggest that the possible rate-limiting step in siRNA delivery is the endosomal escape, which is consistent with the work by Gilleron et al. (2013).

We subsequently developed an optimal control algorithm for gene delivery to take into account the efficacy, toxicity and cell division proposing an important model-based tool for making decisions under uncertainty, which is lacking for gene delivery systems. The proposed modeling and control approach allows for the simulation of siRNA delivery for in vivo conditions in order to compute the optimal delivery profile in the presence of different practical constraints. One of such constraints is on the time to take into account the cell multiplication so that the therapeutic effect is manifested before cell division takes place. 
Cell-doubling effect was addressed to develop a more realistic representation of model-based control of gene delivery that enables to predict the distribution of genetic materials in vivo and before cells divide. A constrained optimization problem was formulated and solved while considering different cell division times to account for uncertainty in cell doubling time. We obtained time profiles of optimal dosage infusion rate and optimal intracellular exposure of siRNAs to exert maximum therapeutic effects. We also demonstrated how these profiles can be affected by the trade-offs between toxicity and efficacy. The multi-objective optimization framework was also set up to control the process system in the presence of other practical constraints such as incremental change in the infusion rate, $\Delta q(t)$, which could be constrained between certain lower and upper bounds. Incorporating of such process constraints is because of the practical limitations of gene delivery devices. In conclusion, the solution of the gene delivery optimal control problem has provided very interesting insights on what siRNA delivery profile might look like in a clinical setting. According to Petrocca and Lieberman (2011), continuous infusion might be used to prolong gene silencing. The results from our study also indicated that an optimal continuous infusion is superior to a bolus injection for achieving maximum gene silencing activity while preserving cell viability. Therefore, a promising platform for gene delivery systems was provided by model-based control technology, which can further assist in the optimization of the process.

Moreover, the potential power of the developed models and control strategy was limited to intracellular barriers due to the lack of suitable experimental data. Model extensions require appropriate experimental analysis that describe spatiotemporal distribution of siRNAs in the extracellular matrix and intracellular environment. Another avenue that can be explored is to build a detailed mathematical model representing the biophysicochemical effects between nanocarriers and biological systems, in order to effectively investigate the intracellular reactions that occur in the cell. Future work involving experimental validation of the 
proposed control profiles will further assist in the development of this technology.

The authors have no conflict of interest to declare. 


\section{References}

Banks GA, Roselli RJ, Chen R, Giorgio TD. 2003. A model for the analysis of nonviral gene therapy. Gene Ther 10(20):1766-1775.

Berraondo P, Gonzalez-Aseguinolaza G, Troconiz IF. 2009. Semi-mechanistic pharmacodynamic modelling of gene expression and silencing processes. Eur J Pharm Sci $37(3-4): 418-426$.

Brooke A, Kendrick D, Meeraus A, Raman R. 1998. GAMS a user's guide. Washington, DC: GAMS Development Corporation.

Bumcrot D, Manoharan M, Koteliansky V, Sah DWY. 2006. RNAi therapeutics: a potential new class of pharmaceutical drugs. Nat Chem Biol 2(12):711-719.

Clark PA, Westerberg AW. 1983. Optimization for design-problems having more than one objective. Comput Chem Eng 7(4):259-278.

Dinh AT, Pangarkar C, Theofanous T, Mitragotri S. 2007. Understanding intracellular transport processes pertinent to synthetic gene delivery via stochastic simulations and sensitivity analyses. Biophys J 92(3):831-846.

Dua P, Dua V, Pistikopoulos EN. 2010. Modelling and multi-parametric control for delivery of anaesthetic agents. Med Biol Eng Comput 48(6):543-553.

Dua V. 2012. Model-based optimal control of non-viral gene delivery. In: Bogle IDL, Fairweather M, editors. 22nd European symposium on computer aided process engineering. Computer Aided Chemical Engineering. Elsevier B.V. 
Dua V, Dua P. 2012. A simultaneous approach for parameter estimation of a system of ordinary differential equations, using artificial neural network approximation. Ind Eng Chem Res 51(4):1809-1814.

Englezos P, Kalogerakis N. 2001. Applied parameter estimation for chemical engineers. New York: Marcel Dekker.

Fire A, Xu SQ, Montgomery MK, Kostas SA, Driver SE, Mello CC. 1998. Potent and specific genetic interference by double-stranded RNA in Caenorhabditis elegans. Nature 391(6669):806-811.

Gabrielsson J, Weiner D. 2010. Pharmacokinetic and pharmacodynamic data analysis: Concepts and applications. Stockholm, Sweden: Swedish Pharmaceutical Press. 247-250 p.

Gilleron J, Querbes W, Zeigerer A, Borodovsky A, Marsico G, Schubert U, Manygoats K, Seifert S, Andree C, Stöter M, Epstein-Barash H, Zhang L, Koteliansky V, Fitzgerald K, Fava E, Bickle M, Kalaidzidis Y, Akinc A, Maier M, Zerial M. 2013. Image-based analysis of lipid nanoparticle-mediated siRNA delivery, intracellular trafficking and endosomal escape. Nat Biotechnol 31(7):638-646.

Horobin RW, Weissig V. 2005. A QSAR-modeling perspective on cationic transfection lipids. 1. predicting efficiency and understanding mechanisms. J Gene Med 7(8):1023-1034.

Jamili E, Dua V. 2016. Modelling and optimal control of non-viral siRNA delivery. In: Kravanja Z, Bogataj M, editors. 26th European symposium on computer aided process engineering, pt A. Computer Aided Chemical Engineering. Elsevier B.V. 38:673-678.

Jandt U, Shao S, Wirth M, Zeng AP. 2011. Spatiotemporal modeling and analysis of transient gene delivery. Biotechnol Bioeng 108(9):2205-2217. 
Kanasty RL, Whitehead KA, Vegas AJ, Anderson DG. 2012. Action and reaction: the biological response to siRNA and its delivery vehicles. Mol Ther 20(3):513-524.

Ledley TS, Ledley FD. 1994. Multicompartment, numerical-model of cellular events in the pharmacokinetics of gene therapies. Hum Gene Ther 5(6):679-691.

Ling XL, Wen L, Zhou Y. 2012. Role of mitochondrial translocation of telomerase in hepatocellular carcinoma cells with multidrug resistance. Int J Med Sci 9(7):545-554.

Lv H, Zhang S, Wang B, Cui S, Yan J. 2006. Toxicity of cationic lipids and cationic polymers in gene delivery. J Control Release 114(1):100-109.

Ma O, Zhang MJ. 2009. Dynamics modeling and control of electroporation-mediated gene delivery. IEEE Trans Autom Sci Eng 6(2):228-238.

Martin TM, Wysocki BJ, Wysocki TA, Pannier AK. 2015. Identifying intracellular pDNA losses from a model of nonviral gene delivery. IEEE Trans Nanobiosci 14(4):455-464.

Martin TM, Wysocki BJ, Beyersdorf JP, Wysocki TA, Pannier AK. 2014. Integrating mitosis, toxicity, and transgene expression in a telecommunications packet-switched network model of lipoplex-mediated gene delivery. Biotechnol Bioeng 111(8):1659-1671.

Nel AE, Madler L, Velegol D, Xia T, Hoek EMV, Somasundaran P, Klaessig F, Castranova V, Thompson M. 2009. Understanding biophysicochemical interactions at the nano-bio interface. Nat Mater 8(7):543-557.

Parker RS, Doyle FJ. 2001. Control-relevant modeling in drug delivery. Adv Drug Delivery Rev 48(2-3):211-228.

Parra-Guillen ZP, Gonzalez-Aseguinolaza G, Berraondo P, Troconiz IF. 2010. Gene therapy: A pharmacokinetic/pharmacodynamic modelling overview. Pharm Res 27(8):1487-1497. 
Petrocca F, Lieberman J. 2011. Promise and challenge of RNA interference-based therapy for cancer. J Clin Oncol 29(6):747-754.

Sandler O, Mizrahi SP, Weiss N, Agam O, Simon I, Balaban NQ. 2015. Lineage correlations of single cell division time as a probe of cell-cycle dynamics. Nature 519(7544):468-471.

Schwake G, Youssef S, Kuhr JT, Gude S, David MP, Mendoza E, Frey E, Radler JO. 2010. Predictive modeling of non-viral gene transfer. Biotechnol Bioeng 105(4):805-813.

Semple SC, Akinc A, Chen JX, Sandhu AP, Mui BL, Cho CK, Sah DWY, Stebbing D, Crosley EJ, Yaworski E, Hafez IM, Dorkin JR, Qin J, Lam K, Rajeev KG, Wong KF, Jeffs LB, Nechev L, Eisenhardt ML, Jayaraman M, Kazem M, Maier MA, Srinivasulu M, Weinstein MJ, Chen QM, Alvarez R, Barros SA, De S, Klimuk SK, Borland T, Kosovrasti V, Cantley WL, Tam YK, Manoharan M, Ciufolini MA, Tracy MA, De Fougerolles A, Maclachlan I, Cullis PR, Madden TD, Hope MJ. 2010. Rational design of cationic lipids for siRNA delivery. Nat Biotechnol 28(2):172-176.

Varga CM, Hong K, Lauffenburger DA. 2001. Quantitative analysis of synthetic gene delivery vector design properties. Mol Ther 4(5):438-446.

Varga CM, Tedford NC, Thomas M, Klibanov AM, Griffith LG, Lauffenburger DA. 2005. Quantitative comparison of polyethylenimine formulations and adenoviral vectors in terms of intracellular gene delivery processes. Gene Ther 12(13):1023-1032.

Videira M, Arranja A, Rafael D, Gaspar R. 2014. Preclinical development of siRNA therapeutics: Towards the match between fundamental science and engineered systems. Nanomed: Nanotechnol, Biol Med 10(4):689-702.

Wang J, Lu Z, Wientjes MG, Au JLS. 2010. Delivery of siRNA therapeutics: Barriers and carriers. Am Assoc Pharm Sci 12(4):492-503. 
Whitehead KA, Langer R, Anderson DG. 2009. Knocking down barriers: Advances in siRNA delivery. Nat Rev Drug Discovery 8(2):129-138.

Williford JM, Wu J, Ren Y, Archang MM, Leong KW, Mao HQ. 2014. Recent advances in nanoparticle-mediated siRNA delivery. Annu Rev Biomed Eng 16:347-370.

Xue HY, Liu S, Wong HL. 2014. Nanotoxicity: A key obstacle to clinical translation of siRNA-based nanomedicine. Nanomed 9(2):295-312.

Yin H, Kanasty RL, Eltoukhy AA, Vegas AJ, Dorkin JR, Anderson DG. 2014. Non-viral vectors for gene-based therapy. Nat Rev Genet 15(8):541-555.

Zhou C, Mao Y, Sugimoto Y, Zhang Y, Kanthamneni N, Yu B, Brueggemeier RW, Lee LJ, Lee RJ. 2012. SPANosomes as delivery vehicles for small interfering RNA (siRNA). Mol Pharmaceutics 9(2):201-210.

Zhou C, Zhang Y, Yu B, Phelps MA, Lee LJ, Lee RJ. 2013. Comparative cellular pharmacokinetics and pharmacodynamics of siRNA delivery by SPANosomes and by cationic liposomes. Nanomed: Nanotechnol, Biol Med 9(4):504-513.

Zhou J, Yockman JW, Kim SW, Kern SE. 2007. Intracellular kinetics of non-viral gene delivery using polyethylenimine carriers. Pharm Res 24(6):1079-1087. 


\section{List of Tables}

1. Table I. Summary of key limitations of previous works.

\section{List of Figures}

1. Figure 1 - Two-compartment model with infusion. A representation of the compartmentalization where $\theta_{i}$ represent the process rate constants: $\theta_{1}$ controls movement out of the endosome and $\theta_{2}$ controls movement from the cytoplasm to the RNAi machinery.

2. Figure 2 - Block diagram for a model-based optimal control of siRNA delivery. Depending on the disease type, the patient output variable of interest could be measured and supplied to a control algorithm.

3. Figure 3 - Pharmacokinetic and pharmacodynamic responses to bolus injections of siRNA therapeutics after start of treatment with total injected doses of $80,100,150$, and $250 \mathrm{nM}$. (a) Time profile of siRNA concentration in the endosome. (b) Time profile of siRNA concentration in the cytoplasm. (c) Time profile of inhibitory effect. (d) Time profile of cell viability.

4. Figure 4 - Trade-off between efficacy and toxicity. A relaxation on $C V^{L O}$ results in a decrease in the minimum total inhibitory effect.

5. Figure 5 - siRNA delivery optimal control results and the pharmacodynamic responses to siRNA infusion over $48 \mathrm{~h}$ transfection for different lower bounds on cell viability when practical limitations of gene delivery devices are not imposed. (a) Time profile of optimal siRNA infusion. (b) Time profile of siRNA concentration in the cytoplasm. (c) Time profile of inhibitory effect. (d) Time profile of cell viability. 
6. Figure 6 - siRNA delivery optimal control results and the pharmacodynamic responses to siRNA infusion over $48 \mathrm{~h}$ transfection when practical limitations of gene delivery devices are imposed. The control optimization problem was solved subject to the system models, and constraints on the cell viability $\left(C V^{L O}=\mathbf{1 0 0} \%\right)$ and the control variable $\left(\boldsymbol{q}^{\max }=\mathbf{3 0}\right.$ or $\left.\mathbf{4 0} \boldsymbol{n M} / \boldsymbol{h}\right)$. (a) Time profile of optimal siRNA infusion. (b) Time profile of siRNA concentration in the cytoplasm. (c) Time profile of inhibitory effect. (d) Time profile of cell viability.

7. Figure 7 - siRNA delivery optimal control results and the pharmacodynamic responses to siRNA infusion over $48 \mathrm{~h}$ transfection when practical limitations of infusion devices are imposed. The optimal control problem was solved subject to the system models, and constraints on the cell viability and the change in infusion rate. (a) Time profile of optimal siRNA infusion. (b) Time profile of siRNA concentration in the cytoplasm. (c) Time profile of inhibitory effect. (d) Time profile of cell viability.

8. Figure 8 - Optimal control results and the pharmacodynamic responses to siRNA infusion over different transfection time periods in order to study the effect of uncertainty in cell division time when practical limitations are not imposed, so $\underline{\mathrm{q}}$ is unconstrained. (a) Time profiles of optimal siRNA infusion for different cell doubling time $\left(T_{d}\right)$ values. (b) siRNA concentration-time profiles in the cytoplasm for different cell doubling time $\left(T_{d}\right)$ values. (c) Percentage inhibitory effect for different cell doubling time $\left(T_{d}\right)$ values. (d) Percentage cell viability for different doubling time $\left(T_{d}\right)$ values. 
9. Figure 9 - Optimal control results and the pharmacodynamic responses to siRNA infusion over different transfection time periods in order to study the effect of uncertainty in cell division time when practical limitations are imposed, so $\underline{q}$ is constrained. (a) Time profiles of optimal siRNA infusion for different cell doubling time $\left(\boldsymbol{T}_{\boldsymbol{d}}\right)$ values. (b) siRNA concentration-time profiles in the cytoplasm for different cell doubling time $\left(\boldsymbol{T}_{\boldsymbol{d}}\right)$ values. (c) Percentage inhibitory effect for different cell doubling time $\left(\boldsymbol{T}_{\boldsymbol{d}}\right)$ values. (d) Percentage cell viability for different doubling time $\left(\boldsymbol{T}_{\boldsymbol{d}}\right)$ values. 


\section{Tables}

Table I. Summary of key limitations of previous works.

\begin{tabular}{|c|c|}
\hline \multicolumn{1}{|c|}{ Limitations } & References \\
\hline Toxicity of vectors was left unaccounted for. & Varga et al., 2001, 2005; Banks et al., 2003; \\
\hline Effects of cell division were not & Dinh et al., 2007; Jandt, 2011 \\
\hline incorporated. & Varga et al., 2001, 2005; Banks et al., 2003; \\
\hline Unable to predict transfection for in vivo & Binh et al., 2007 \\
\hline applications. & \\
\hline Effect of uncertainty in cell doubling time & Varga et al., 2001, 2005; Banks et al., 2003; \\
\hline was not addressed. & Dinh et al., 2007; Martin et al, 2015 \\
\hline
\end{tabular}




\section{Figures}

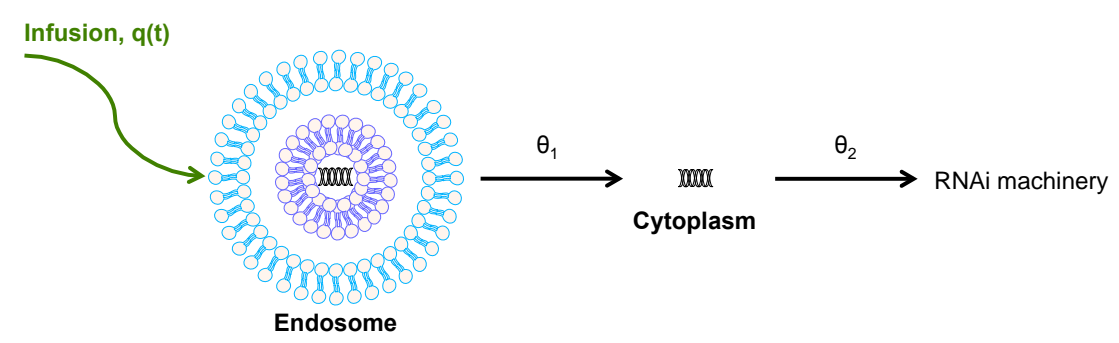

Figure 1 - Two-compartment model with infusion. A representation of the compartmentalization where $\theta_{i}$ represent the process rate constants: $\theta_{1}$ controls movement out of the endosome and $\theta_{2}$ controls movement from the cytoplasm to the RNAi machinery.

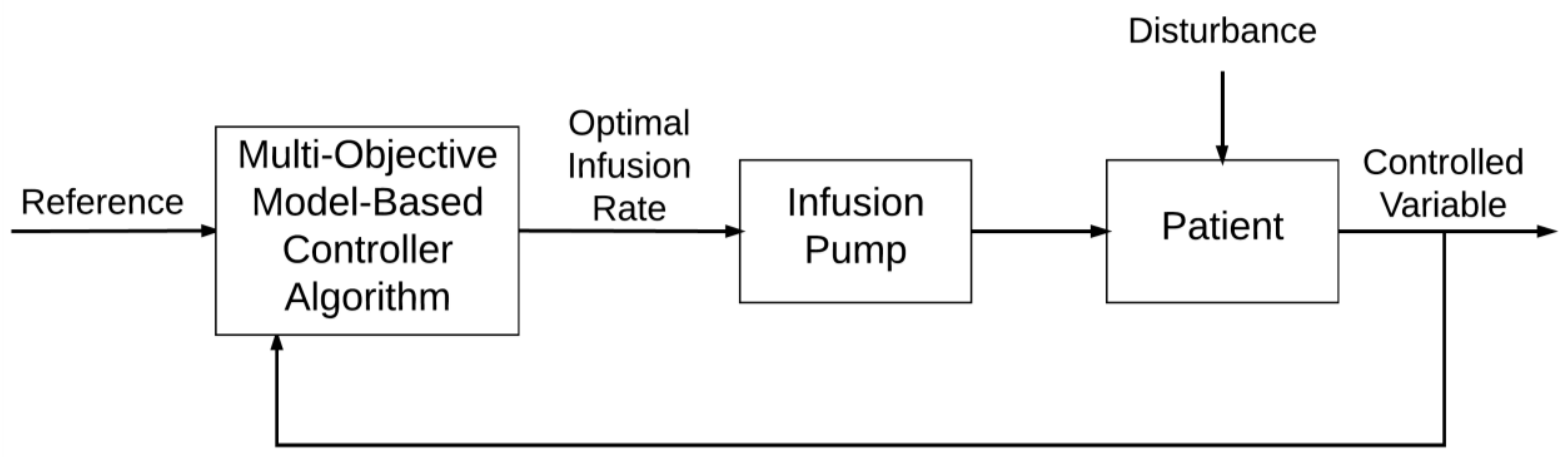

Figure 2 - Block diagram for a model-based optimal control of siRNA delivery. Depending on the disease type, the patient output variable of interest could be measured and supplied to a control algorithm. 

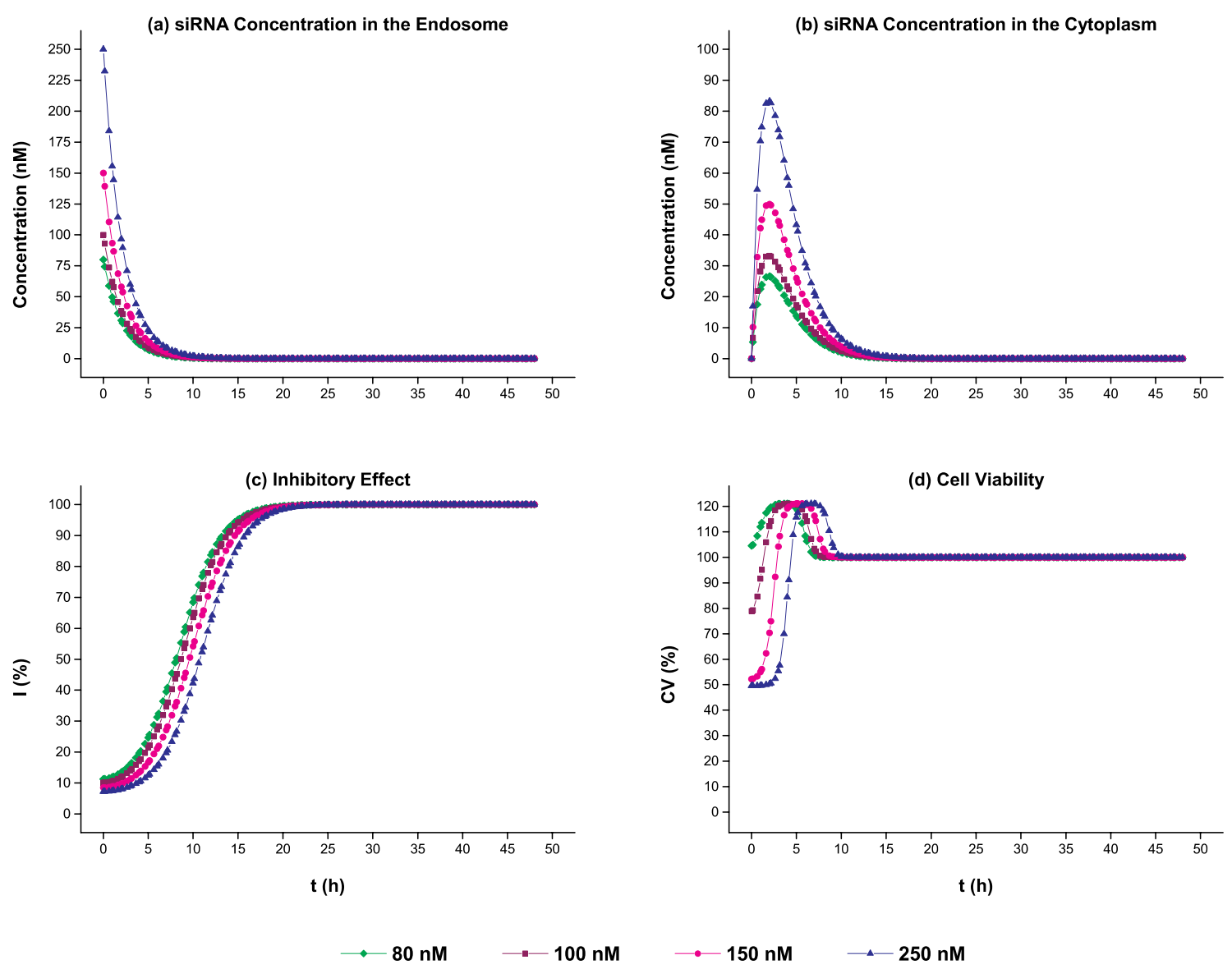

Figure 3 - Pharmacokinetic and pharmacodynamic responses to bolus injections of siRNA therapeutics after start of treatment with total injected doses of $80,100,150$, and $250 \mathrm{nM}$. (a) Time profile of siRNA concentration in the endosome. (b) Time profile of siRNA concentration in the cytoplasm. (c) Time profile of inhibitory effect. (d) Time profile of cell viability. 


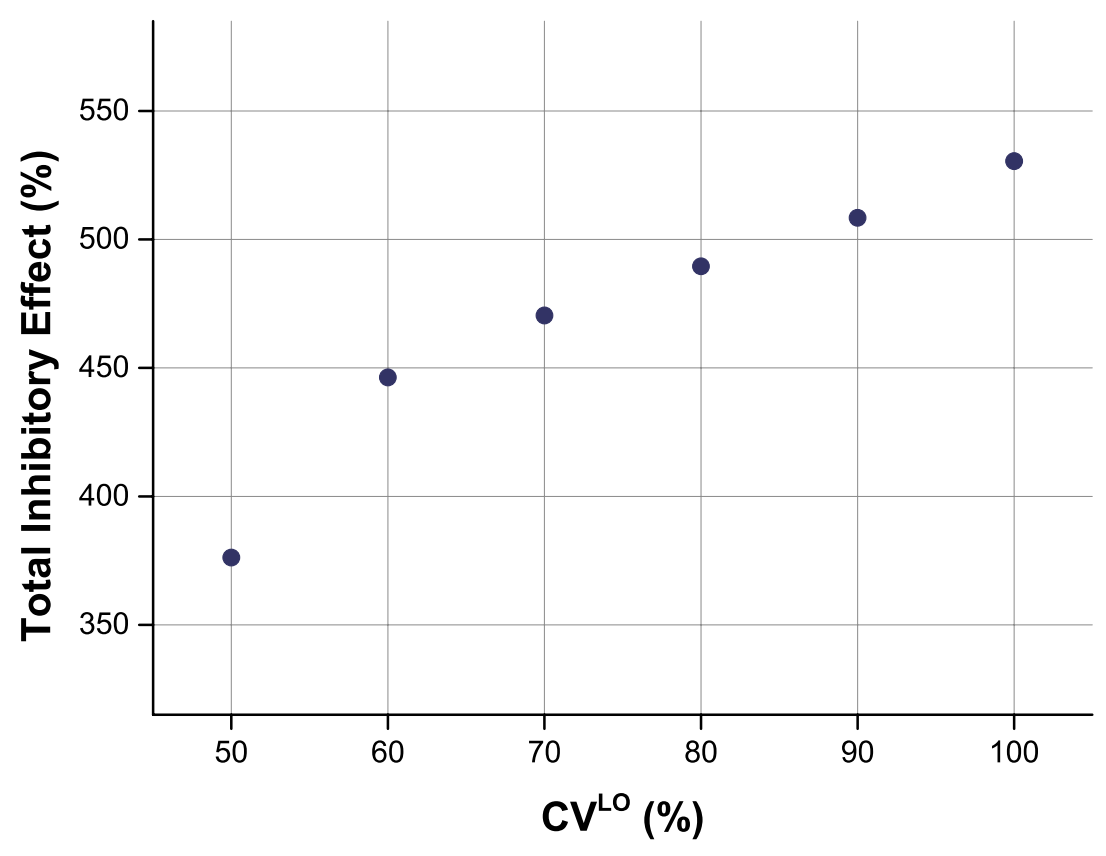

Figure 4 - Trade-off between efficacy and toxicity. A relaxation on $C V^{L O}$ results in a decrease in the minimum total inhibitory effect. 

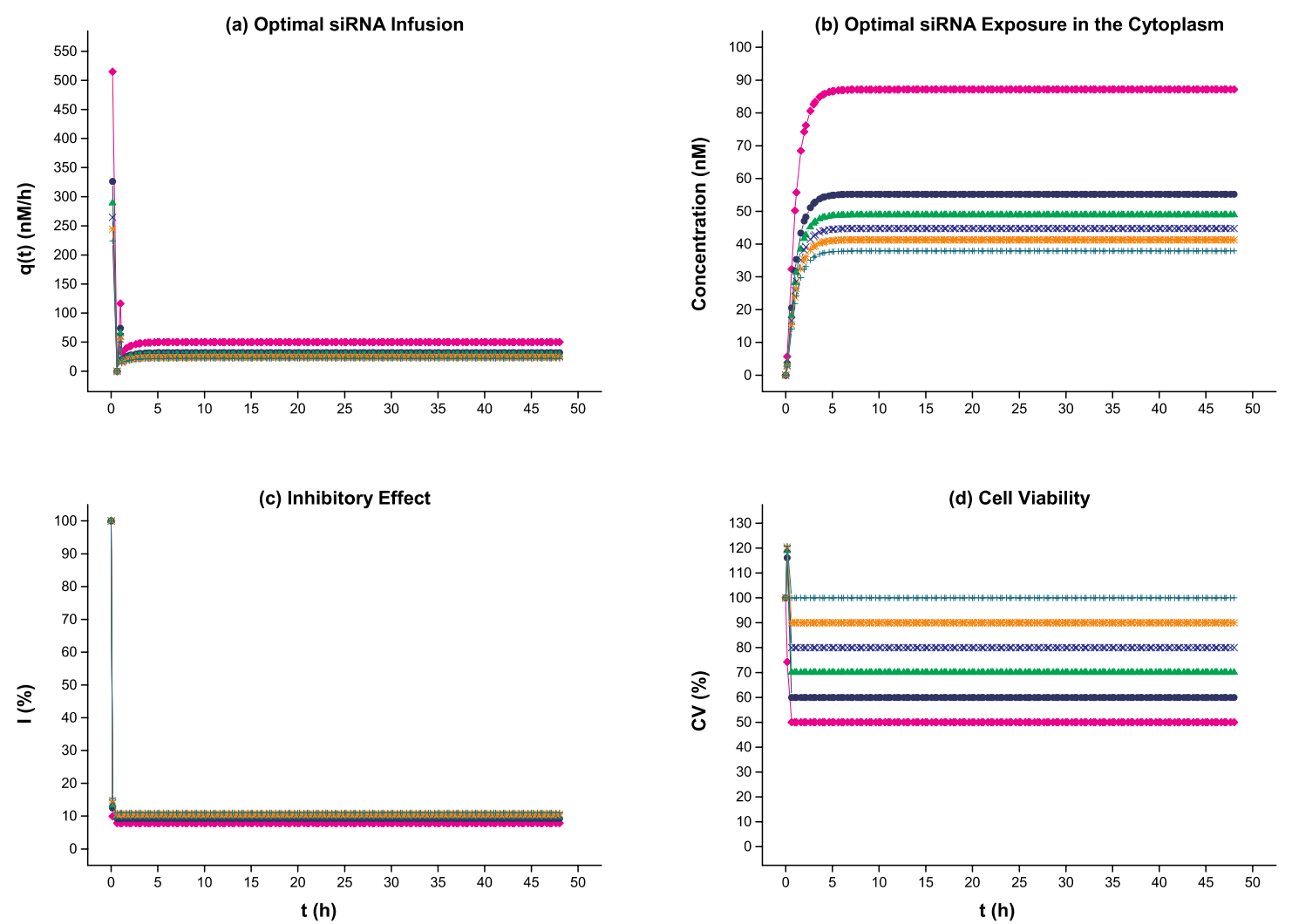

$\longrightarrow$ CV.LO $=50 \% \quad \longrightarrow$ CV.LO $=60 \% \quad \longrightarrow$ CV.LO $=70 \%$

CV.LO $=80 \%$

CV.LO $=90 \%$

CV.LO $=100 \%$

Figure 5 - siRNA delivery optimal control results and the pharmacodynamic responses to siRNA infusion over 48 h transfection for different lower bounds on cell viability when practical limitations of gene delivery devices are not imposed. (a) Time profile of optimal siRNA infusion. (b) Time profile of siRNA concentration in the cytoplasm. (c) Time profile of inhibitory effect. (d) Time profile of cell viability. 

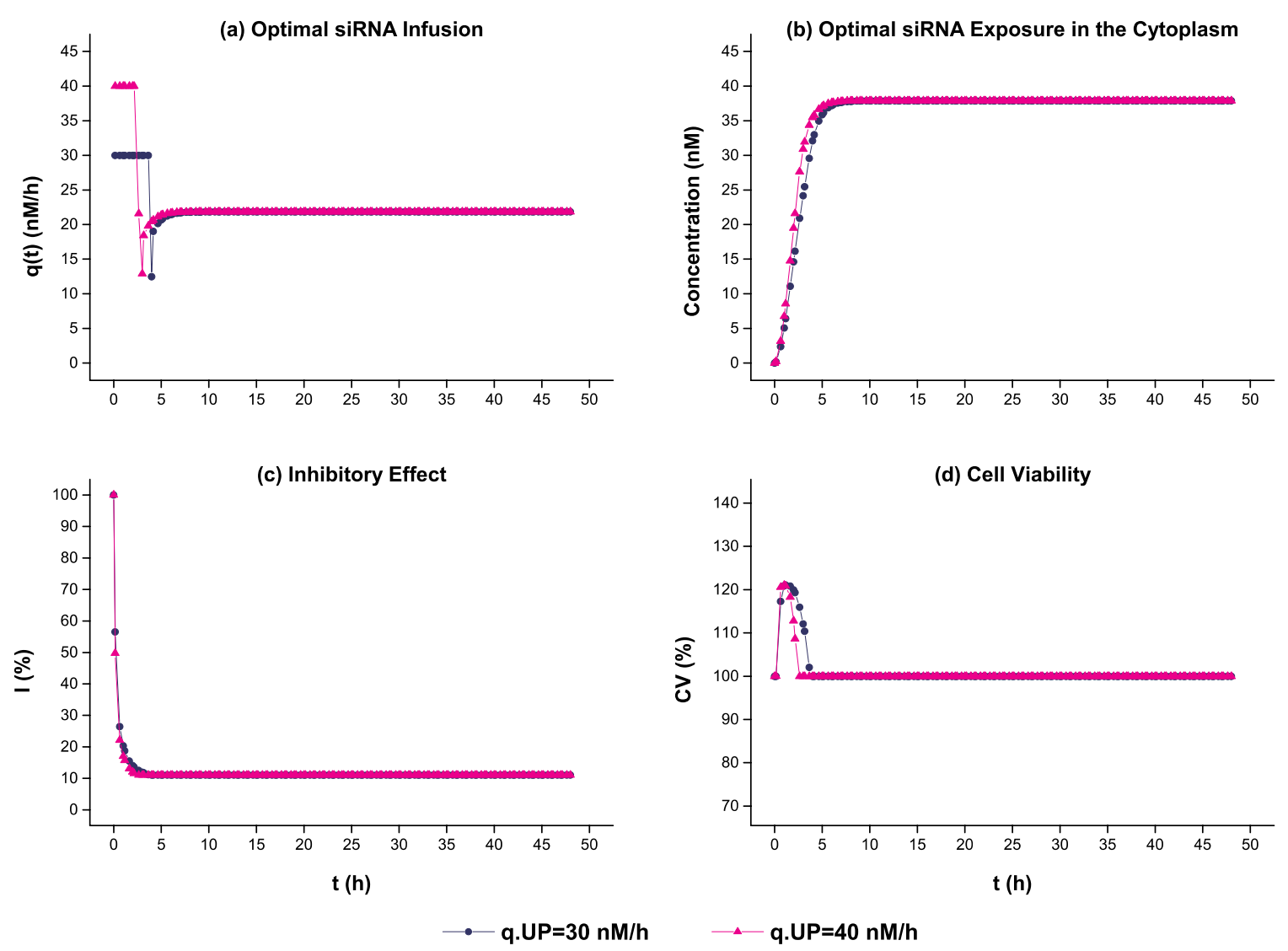

Figure 6 - siRNA delivery optimal control results and the pharmacodynamic responses to siRNA infusion over $48 \mathrm{~h}$ transfection when practical limitations of gene delivery devices are imposed. The control optimization problem was solved subject to the system models, and constraints on the cell viability $\left(C V^{L O}=100 \%\right)$ and the control variable $\left(q^{\max }=30\right.$ or $\left.40 n M / h\right)$. (a) Time profile of optimal siRNA infusion. (b) Time profile of siRNA concentration in the cytoplasm. (c) Time profile of inhibitory effect. (d) Time profile of cell viability. 

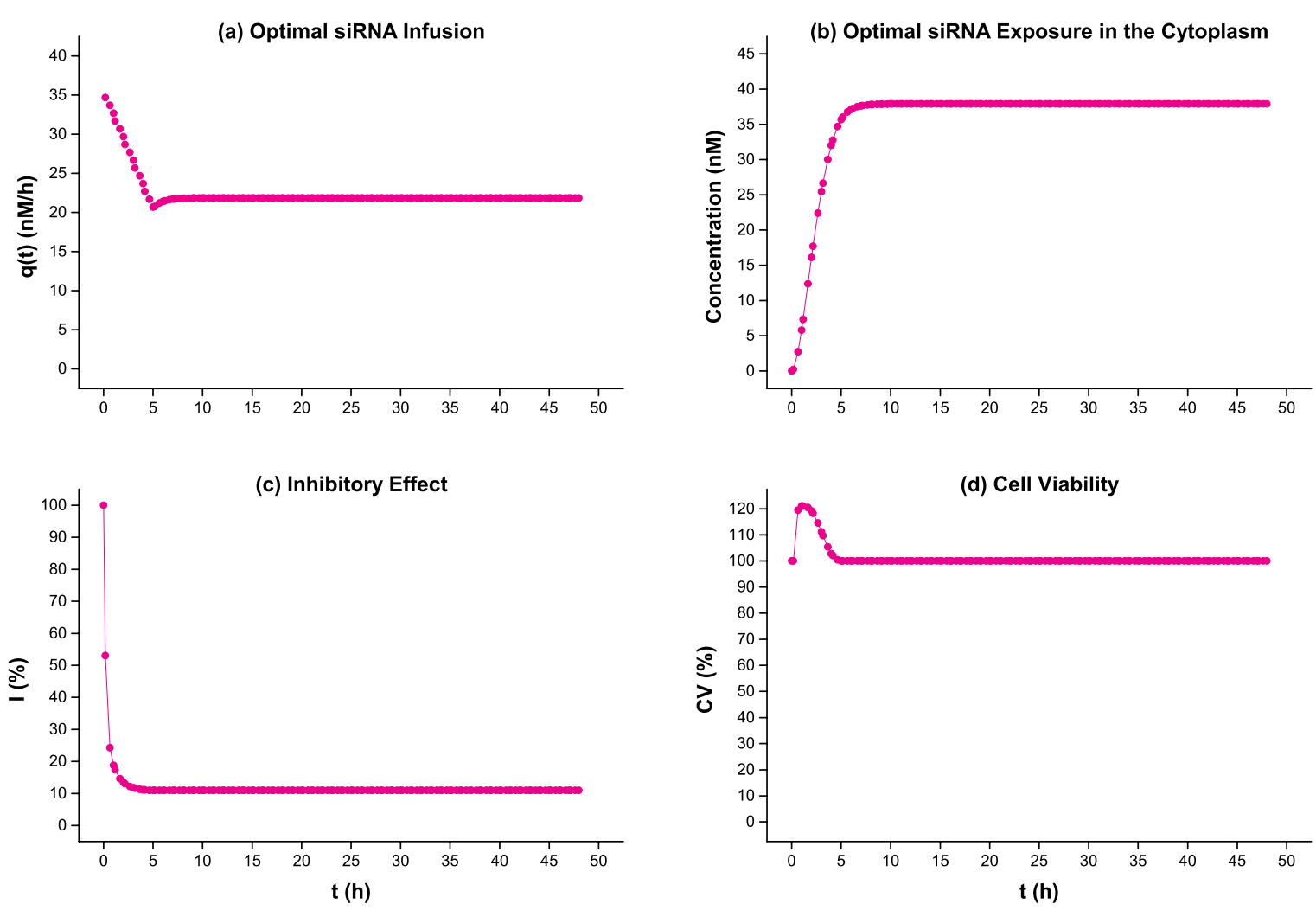

Figure 7 - siRNA delivery optimal control results and the pharmacodynamic responses to siRNA infusion over $48 \mathrm{~h}$ transfection when practical limitations of infusion devices are imposed. The optimal control problem was solved subject to the system models, and constraints on the cell viability and the change in infusion rate. (a) Time profile of optimal siRNA infusion. (b) Time profile of siRNA concentration in the cytoplasm. (c) Time profile of inhibitory effect. (d) Time profile of cell viability. 

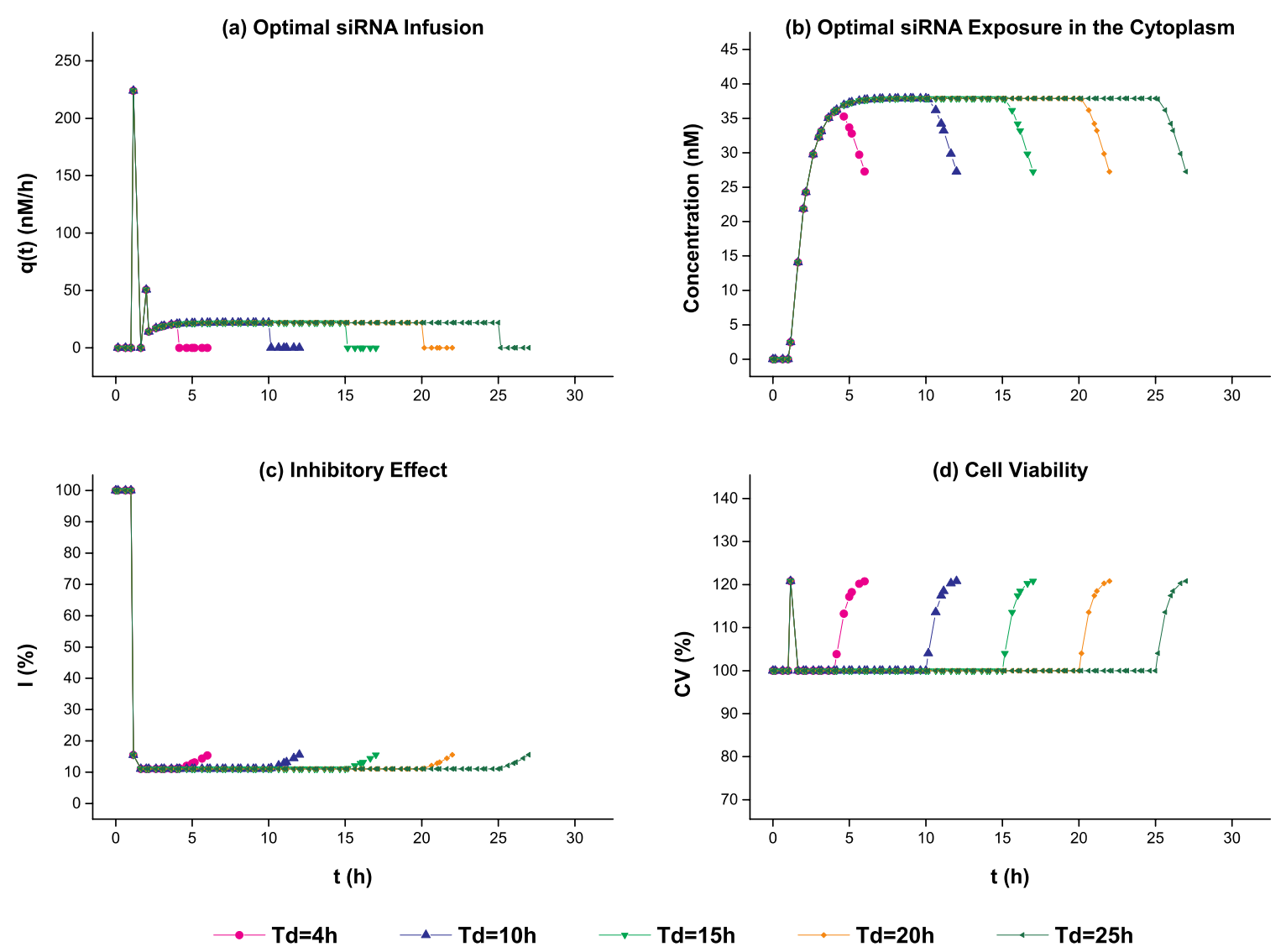

Figure 8-Optimal control results and the pharmacodynamic responses to siRNA infusion over different transfection time periods in order to study the effect of uncertainty in cell division time when practical limitations are not imposed, so $\underline{q}$ is unconstrained. (a) Time profiles of optimal siRNA infusion for different cell doubling time $\left(T_{d}\right)$ values. (b) siRNA concentration-time profiles in the cytoplasm for different cell doubling time ( $\left.T_{d}\right)$ values. (c) Percentage inhibitory effect for different cell doubling time $\left(T_{d}\right)$ values. (d) Percentage cell viability for different doubling time $\left(T_{d}\right)$ values. 

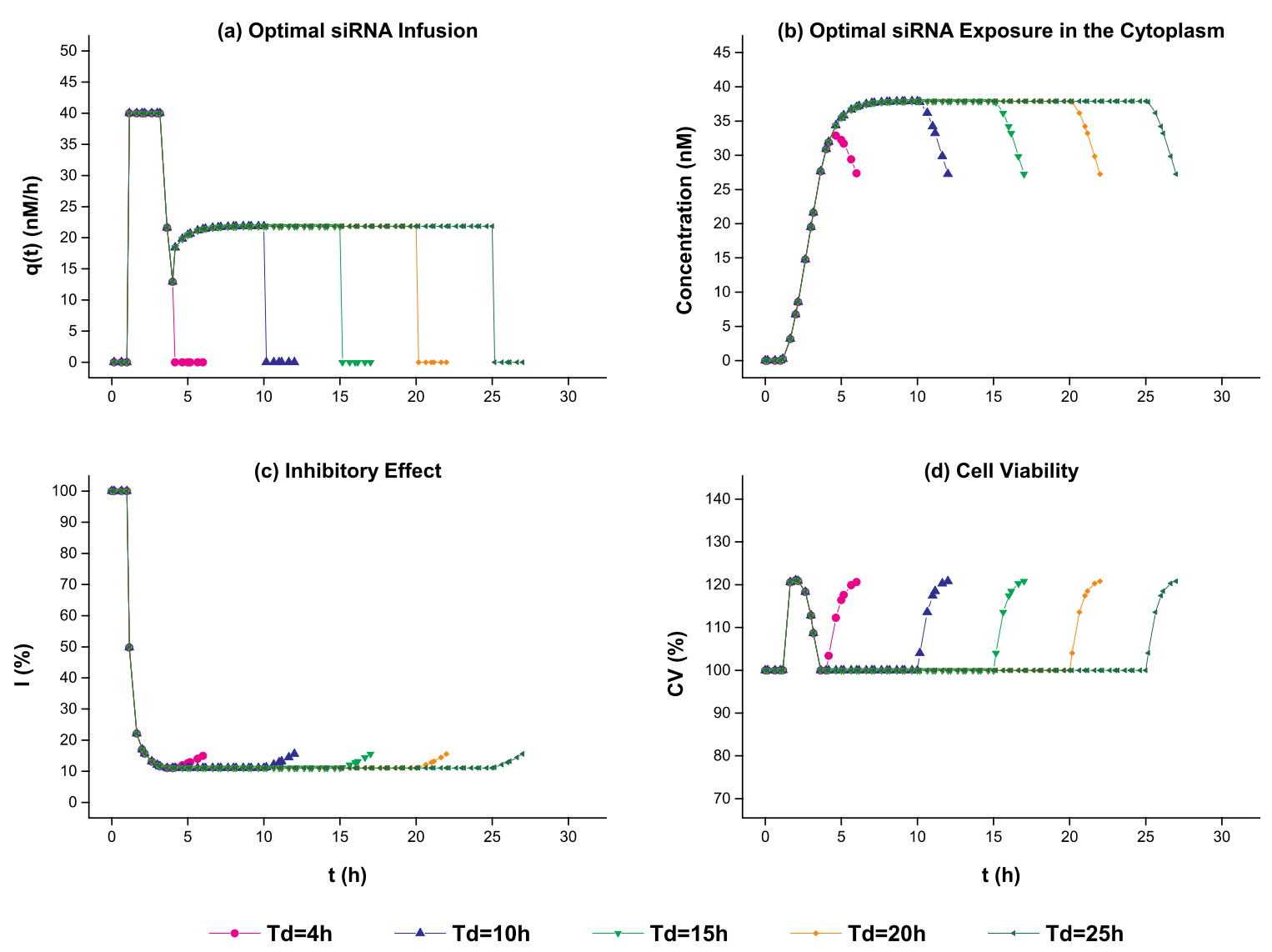

Figure 9 - Optimal control results and the pharmacodynamic responses to siRNA infusion over different transfection time periods in order to study the effect of uncertainty in cell division time when practical limitations are imposed, so $\underline{q}$ is constrained. (a) Time profiles of optimal siRNA infusion for different cell doubling time $\left(T_{d}\right)$ values. (b) siRNA concentration-time profiles in the cytoplasm for different cell doubling time $\left(T_{d}\right)$ values. (c) Percentage inhibitory effect for different cell doubling time $\left(T_{d}\right)$ values. (d) Percentage cell viability for different doubling time $\left(T_{d}\right)$ values. 